\title{
Correlating seismic wave velocity measurements with mining activities at Williams Mine
}

DB Rebuli Institute of Mine Seismology, Canada

\begin{abstract}
Seismic wave velocities are sensitive to rock mass properties including fracture density, orientation, stress magnitude and orientation. By measuring small changes, of the order of $0.01 \%$, in the seismic wave velocities over time, it is possible for track changes in the rock mass properties to occur. In mining, the rock mass properties are altering as the rock is removed or pillars are formed. By tracking these changes, it may be possible to get a better understanding of the effect mining has on the rock mass properties. These changing rock mass properties are important for reliable static stress modelling of the mine and for stability analysis. We use an active seismic source where a pneumatic hammer generates repeatable impacts that can be measured a few hundred metres away from the source. This allows us to measure directional velocity changes where these can be compared with mining and microseismic activity. This is the first step in understanding the effect of mining on the rock mass properties over time.
\end{abstract}

Keywords: seismic monitoring, active seismic source, velocity changes

\section{Introduction}

Seismic monitoring networks have become more common in mining environments, especially in deep underground mines where seismic activity and rockfalls can be significant hazards. The need for geotechnical engineers to monitor the rock mass response to mining over time is important since most seismic events in mines are induced by mining activities. Seismic monitoring in mines has made great progress as a tool to analyse some of the risks involved in mining at depth, but this does not always give a good representation of how the rock mass is responding.

Seismic velocities of intact rock are sensitive to the internal state of the rock mass. Changes in applied stress (Larose \& Hall 2009), pore pressure (Brenguier et al. 2014) and fracture density (Nur \& Simmons 1969; Nur 1971) have all been shown to cause measurable changes in seismic velocity. There have been several investigations over the years to try to understand time-dependent seismic velocity variations and how this relates to stress. Some results showing velocity variations prior to earthquakes have been reported in the literature (for example Niu et al. 2008), but more research is needed to verify the efficacy of seismic velocity variations as a reliable precursor to earthquakes.

One method of measuring seismic wave velocity changes uses passive seismic data for double-difference tomography (Zhang \& Thurber 2003). Although, when using passive seismic sources, there is no control over the temporal resolution of the measurements (when earthquakes occur) or spatial distribution of sources (where they occur).

As a means to better control the temporal and spatial distribution, different types of controlled man-made seismic sources have been used to produce repetitive waveforms to measure seismic velocity variations (Fazio et al. 1973; Reasonberg \& Aki 1974; Yamamura et al. 2003). Since the initiation time and location of the seismic signal are controllable, the measurements can be made routinely (Lynch et al. 2013) and velocities variations of the order of $0.01 \%$ are possible. The limitation is that the range of these sources can be short, depending on the type of source used. For example, for high-frequency sources, the signal is attenuated over shorter distances, compared with lower frequency sources. 
In this paper, an active seismic source was used to monitor seismic velocity variations in an area of the Williams Mine in Canada. Accurate monitoring of these velocity variations with time may provide information on the rock mass response to mining activities. A background to the geology at Williams Mine can be found in early work by Earl et al. (2015).

\section{$2 \quad$ Method}

A pneumatic seismic source was installed in a short borehole in the mine near a sill pillar which was being mined. The borehole was drilled pointing towards the sill pillar of interest at an angle of about $15^{\circ}$ up from horizontal. High-pressure air was used to propel a $3 \mathrm{~kg}$ stainless steel mass within a $2 \mathrm{~m}$ tube to create an impact against a solid surface at the end. A $14 \mathrm{~Hz}$ geophone was installed just below the source to act as a reference signal. The source was set to fire every $15 \mathrm{~s}$ to produce a repeatable signal from a fixed location. Due to the sensor configuration, it was required that the signal be recorded on geophones between 100 and $300 \mathrm{~m}$ away from the active source. While driving the source at a pressure of $200 \mathrm{kPa}$, a signal could be detected up to $190 \mathrm{~m}$ away, although this signal was small in amplitude. This placed a limitation on the smallest time shift that could be extracted when comparing two similar but time-shifted signals. This time shift is related to the signal-to-noise (SNR) ratio by the Cramer-Rao lower bound (Kay 1993; Silver et al. 2007):

$$
\sigma \geq \frac{1}{2 \pi \times f_{0} \times S N R}
$$

where:

$$
\begin{aligned}
& \sigma=\text { time shift, } f_{0} \text { is the dominant frequency of the signal. } \\
& S N R=\text { signal-to-noise ratio. }
\end{aligned}
$$

Since the active source produces a highly repeatable signal, the recorded signal can be stacked over time to increase the SNR ratio. An example of the improved SNR ratio of a stacked signal compared with a single measurement is shown in Figure 1. The stack was created using one hour's worth of signals, although not every impact was used. This sensor was about $30^{\circ}$ up from the active source, which is ideal for recording both $\mathrm{P}$ and $\mathrm{S}$-waves from the impact.

Site 32 (176 m away)

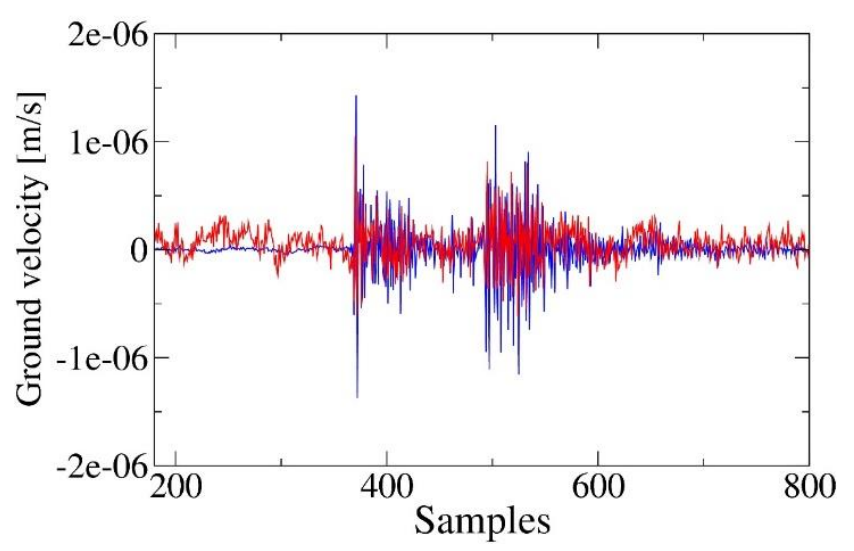

Figure 1 Waveforms from a single shot (red) and one hour stack (blue) for sensor 32 near the sill pillar. The noise has been significantly reduced by stacking 
Figure 2 shows an hour stack compared with a single shot for another sensor. This sensor is almost perpendicular to the direction of the source impact, resulting in strong $\mathrm{S}$-waves arriving at this sensor but only small P-waves. Geological features which give a velocity contrast can result in P-waves being created from the impinging S-waves, but these would be much smaller in amplitude and their arrival times would depend on the distances between the feature, the active source and the sensor. For the case of sensor 40, the time of the small P-arrival is consistent with the direct P-wave from the source.

Site 40 (170 m away)

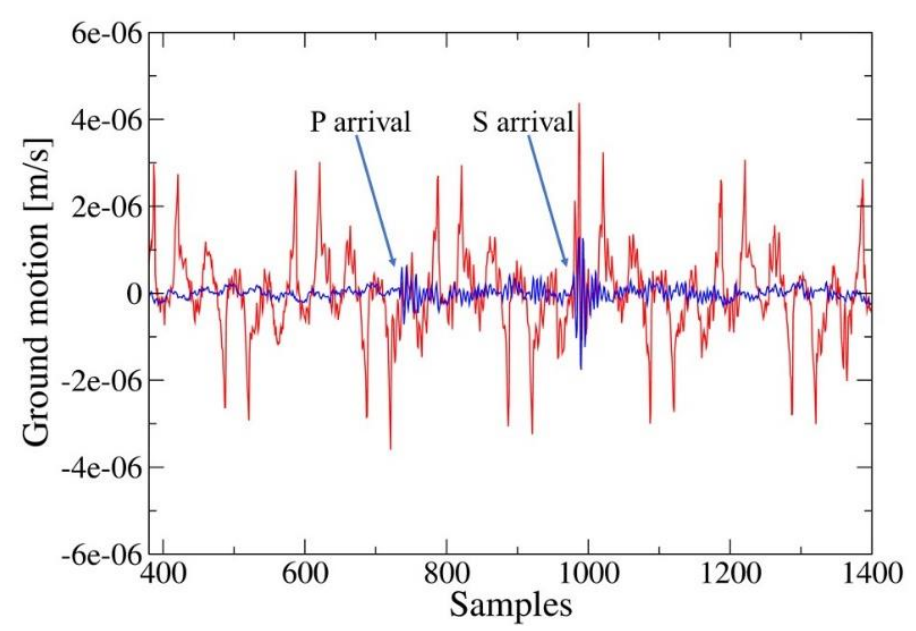

Figure 2 Waveforms from a single shot (red) and one hour stack (blue) for sensor 40 which is almost perpendicular to the active source. A strong S-wave arrival is observed and the noise is significantly reduced by stacking. A small P-wave arrival is visible after stacking and both arrivals are indicated on the plot

Since the time delay variations measured are significantly smaller than the sampling period afforded by the data acquisition system (around $100 \mu \mathrm{s}$ ), the time delay can either be determined using significantly up-sampled signals or by measuring the phase shift between signals. The up-sampling method is straightforward but comes with significantly increased processing time. The phase shift method, although more complex, is computationally quick and was used in this analysis.

The signals from the hourly stacks were compared to the reference signal to calculate the relative time shifts for a selection of sensor positions.

\section{Data analysis}

The layout of the sensors near the sill pillar is shown in Figure 3. Here, site 96 is the reference sensor, site 32 is the closest sensor to the pillar, and site 40 is far from any mining activity for the time period considered. A two-week period was taken in February 2016. A plot of significant seismicity is shown in Figure 4. Unfortunately for the experiment, the seismicity located a little to the west of the sensor, but this was close enough such that the influence of the seismicity on local stress conditions was measurable. Unfortunately, the air hose powering the active source was disconnected on 24 February, so there is only data from the active source for a few days, while there was seismicity in the area. 




(a)

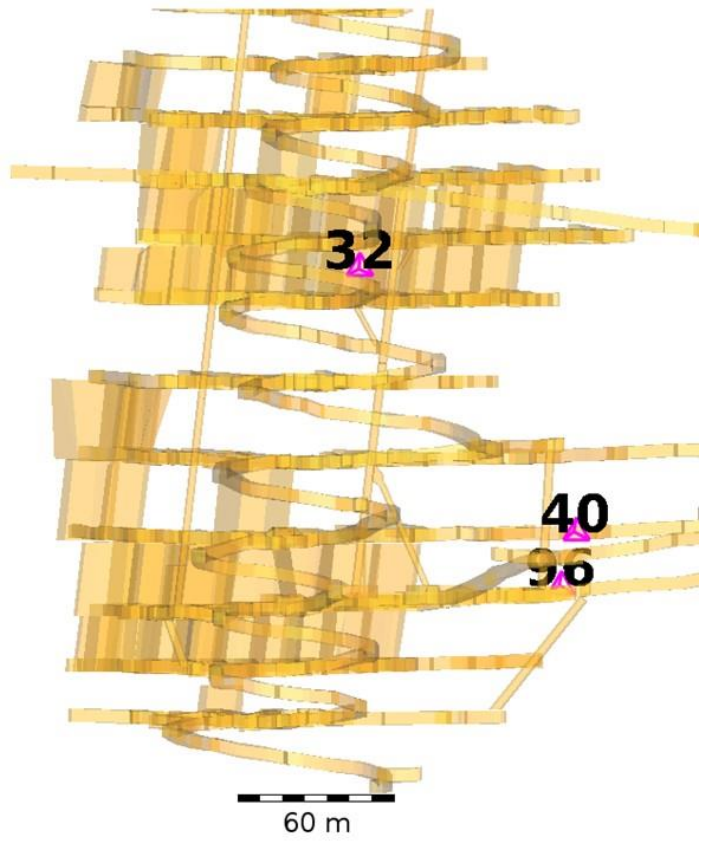

(b)

Figure 3 Sensor layout looking east (a); and looking north (b). Site 96 is the reference sensor, while site 32 is the closest to the sill pillar. Site $\mathbf{4 0}$ is away from mining activity and seismicity, and was useful as a control



Figure 4 Plot (looking north) of the significant seismicity recorded during the period of interest. These events ranged from $\mathrm{m} 0.1$ to $\mathrm{m} 0.4$

Since site 32 could capture both the P and S-waves from the active source, the velocity variations of both waves can be compared with seismicity and mining activities. Figure 5 shows the $\mathrm{P}$ and S-wave velocity variations. The P-wave velocity variation curve shows an increase in the velocity in response to an m0.2 event in the early hours of the morning of 19 February. The P-wave velocity then decreased in response to an $\mathrm{m} 0.3$ event recorded in the afternoon of 19 February. The S-wave velocity shows a similar trend, although this decrease started a little later. Both velocities show a response to another $\mathrm{m} 0.3$ event recorded in the early hours of 20 February. Again, both velocities increase around the time of an m0.1 event just before midday on 20 February, although the S-wave velocity increases significantly more 
than the P-wave velocity. The red dashed line in Figure 4 is the time of a blast in the area. Both velocities start to decrease in response to this blast, although this time the P-wave response is stronger. The S-wave velocity shows a slow downward trend from this point on while the P-wave velocity does respond to a few more significant events in the afternoon of 21 February. The grey line in Figure 4 shows the S-wave velocity curve for site 40 . This shows very little response, which is to be expected as this site is located away from the seismicity and mining activities.



Figure 5 Velocity variation curves for the period of interest for the P-wave (blue) and S-wave (green) for site 32. The S-wave velocity variations recorded at site 40 are shown in grey. The dashed vertical lines indicate times of significant seismic events (as shown in Figure 4) while the red dashed line indicates the time of the blast. The labelled date indicators are for midday of each day

Since site 32 is a triaxial sensor, it may be of interest to look at the velocity variations for each component, keeping in mind that this sensor is almost aligned with the direction of the active source and is above the source. Figure 5 shows the P-wave velocity variations, while Figure 6 shows the S-wave velocity variations. All three components show similar P-wave variations, and the $\mathrm{z}$-component of the $\mathrm{S}$-wave follows a similar trend. The horizontal components of the S-wave show a different trend. The x-axis of the sensor is coincidently almost perfectly aligned with sigma 1 .

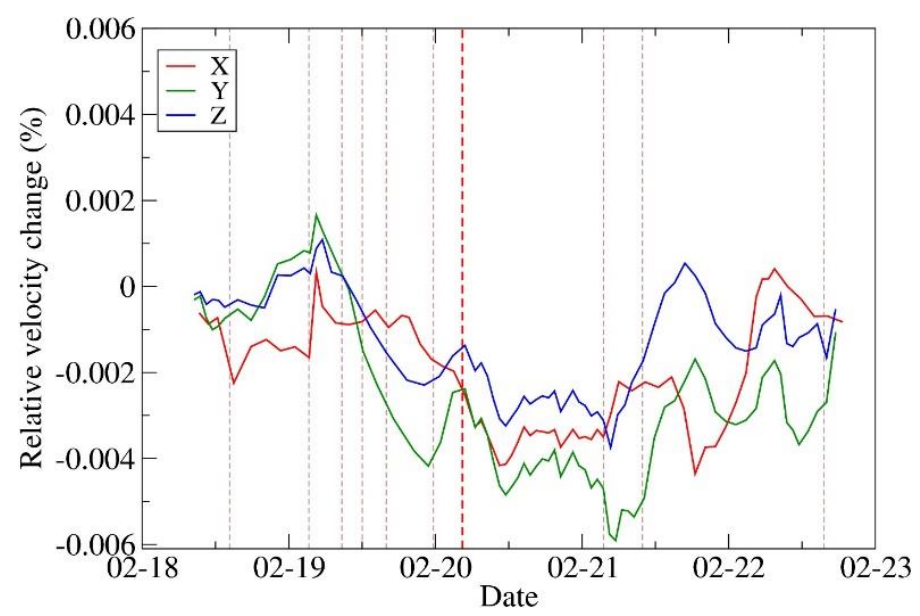

Figure 6 P-wave velocity variation curves for the period of interest for $x$ (red), y (green) and $z$ (blue) sensor components for site 32. The dashed vertical lines indicate times of significant seismic events (as shown in Figure 4) while the red dashed line indicates the time of the blast. The labelled date indicators are for midday of each day 
Velocity variations in P-waves are sensitive to stress changes in the direction parallel to the direction of wave propagation, while variations in the S-wave velocity are sensitive to stress changes perpendicular to the wave propagation. For this set-up, with site 32 mostly vertically above the active source, the variations in the P-wave velocity, which should be mostly picked up by the z-component, should be sensitive to changes in the vertical stress. The variations in the S-wave velocity, along the horizontal sensor components, should be sensitive to horizontal stress changes.

Figure 7 suggests the vertical stress decreases from the afternoon of 19 February, but starts to recover in the afternoon of 21 February. The z-component of the S-wave velocity curve follows a similar trend. It is interesting to note that the two horizontal components of the S-wave start off differently, but by the early morning of 20 February these follow a very similar trend, suggesting an increase in the horizontal stress peaking at the time of the blast on 20 February. The horizontal stress then decreases until the air pressure on the active source was accidentally switched off. The changes in the horizontal stresses are almost opposite to the changes in the vertical stress for the period leading up to the blast. They then follow a similar trend until the time of the significant events in the afternoon of 21 February, before diverging again.

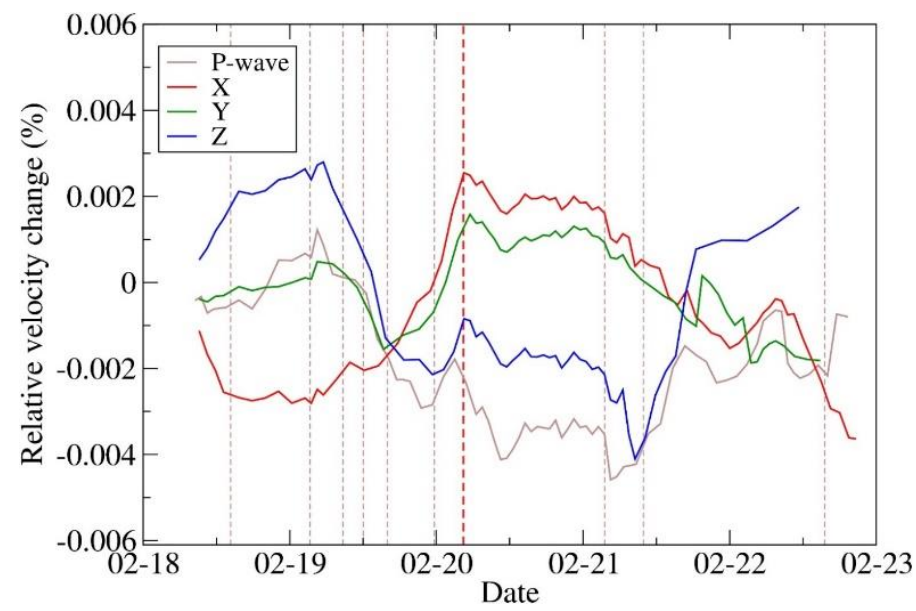

Figure 7 S-wave velocity variation curves for the period of interest for $x$ (red), y (green) and $z$ (blue) sensor components for site 32 . The solid grey line is the average $P$-wave velocity variation for site 32. The dashed vertical lines indicate times of significant seismic events (as shown in Figure 4) while the red dashed line indicates the time of the blast. The labelled date indicators are for midday of each day

\section{Conclusion}

A pneumatic source was installed at Williams Mine near a sill pillar of interest. The repeating nature of the source allowed the recorded signals at a couple of sensors, about $170 \mathrm{~m}$ away, to be stacked. The stacking was successful in increasing the SNR to the point where seismic wave velocity variations of the order of $0.001 \%$ could be measured. This was sufficient to pick up changes in the velocities at a sensor near the sill pillar while mining was underway.

The changes correlated well with significant seismic events in the sill pillar and with a blast taken on one of the days while the pneumatic source was operating. A sensor at a similar distance from the source, but away from mining, was also monitored and showed very little in the way of velocity changes. This indicates that the changes observed on the sensor near the sill pillar are not related to regional changes, but rather local changes associated with the mining of the sill pillar.

Modelling the stress changes as mining progressed for this period is the next step in understanding the velocity changes observed. This will give insight into how the orientations of the joint sets and other local geological features affect the velocity changes, as microfractures are opened or closed. 
The velocity variations for the $\mathrm{P}$ and $\mathrm{S}$-waves at the sensor of interest showed different behaviours during the period of monitoring. This suggests some anisotropy in the stress changes around the sensor during this time. This may be related to the orientations of the joint sets, but further work is necessary to confirm this.

The pneumatic source is a useful tool for measuring small seismic wave velocity changes in a mining environment. The velocity changes can be correlated with mining activities, and one of the next steps is to monitor a sensor recently installed in the sill pillar to see if stress changes before a large seismic event can be detected.

\section{Acknowledgement}

The author thanks Williams Mine for hosting the active seismic source experiment, for their help in installing and maintaining the equipment, and for allowing this work to be presented. The author also thanks the Ultra Deep Mining Network (UDMN) program for supporting this project.

\section{References}

Brenguier, F, Campillo, M, Takeda, T, Aoki, Y, Shapiro, N, Briand, X, Emoto, K \& Miyake, H 2014, 'Mapping pressurized volcanic fluids from induced crustal seismic velocity drops', Science, vol. 345, pp. 80-82.

Earl, PJ, Malovichko, D \& Rebuli, D 2015, 'Study of stress conditions at Williams Mine using underground observations and microseismic monitoring data', in $\mathrm{Y}$ Potvin (ed.), Proceedings of the International Seminar on Design Methods in Underground Mining, Australian Centre for Geomechanics, Perth, pp. 149-163.

Fazio, TD, Aki, L \& Alba, K 1973, 'Solid earth tide and observed change in the in situ seismic velocity', Journal of Geophysical Research, vol. 78, pp. 1,319-1,322.

Kay, SM 1993, Fundamentals of Statistical Signal Processing, Volume 1: Estimation theory, Prentice Hall, Englewood Cliffs.

Larose, E \& Hall, S 2009, 'Monitoring stress related velocity variation in concrete with a $2 \times 10^{-5}$ relative resolution using diffuse ultrasound', Journal of the Acoustical Society of America, vol. 125, pp. 1,853-1,856.

Lynch, RAL, Olivier, G \& Green, M 2013, 'High accuracy measurements of seismic velocity variations in mines', A Malovichko \& D Malovichko (eds), Proceedings of the 8th International Symposium of Rockbursts and Seismicity in Mines, Geological Survey of the Russian Academy of Sciences, Moscow, pp. 157-165.

Niu, F, Silver, PG, Daley, TM, Cheng, X \& Majer, EL 2008, 'Preseismic velocity changes observed from active source monitoring at the Parkfield SAFOD drill site', Nature, vol. 454, pp. 204-208.

Nur, A 1971, 'Effects of stress on velocity anisotropy in rocks with cracks', Journal of Geophysical Research, vol. 76, pp. 2,022-2,034.

Nur, A \& Simmons, G 1969, 'Stress-induced velocity anisotropy in rock: An experimental study', Journal of Geophysical Research, vol. 74, pp. 6667-6674.

Reasonberg, P \& Aki, K 1974, 'A precise, continuous measurement of seismic velocity for monitoring in situ stress', Journal of Geophysical Research, vol. 79, pp. 399-406.

Silver, PG, Daley, TM, Nui, F \& Majer, EL 2007, 'Active source monitoring of cross-well seismic travel time for stress-induced changes', Bulletin of the Seismological Society of America, vol. 97, pp. 281-293.

Yamamura, K, Sano, O, Utada, H, Takei, Y \& Nakao, S 2003, 'Long-term observation of in situ seismic velocity and attenuation', Journal of Geophysical Research, vol. 108, pp. 2,317-2,331.

Zhang, H \& Thurber, CH 2003, 'Double-difference tomography: the method and its application to the Hayward fault, California', Bulletin of the Seismological Society of America, vol. 93, pp. 1,875-1,889. 
